\title{
(2) OPEN ACCESS \\ Are disruptive innovations recognised in the healthcare literature? A systematic review
}

\author{
Viknesh Sounderajah (10,1,2 Vanash Patel, ${ }^{1,3}$ Lavanya Varatharajan, ${ }^{4}$ \\ Leanne Harling, ${ }^{1,5}$ Pasha Normahani, ${ }^{1}$ Joshua Symons, ${ }^{1}$ James Barlow, ${ }^{6}$ \\ Ara Darzi, ${ }^{1,2}$ Hutan Ashrafian ${ }^{1,2}$
}

- Additional material is published online only. To view, please visit the journal online (http://dx.doi.org/10.1136/ bmjinnov-2020-000424).

${ }^{1}$ Department of Surgery and Cancer, Imperial College London, London, UK Institute of Global Health Innovation, Imperial College London, London, UK

${ }^{3}$ Department of General Surgery, Watford General Hospital, West Hetfordshire NHS Trust, Watford, UK

${ }^{4}$ Department of Bariatric

Surgery, Ashford and St. Peter's Hospitals NHS Foundation Trust, Chertsey, UK

${ }^{5}$ Department of Thoracic Surgery,

Guy's and St Thomas' NHS Foundation Trust, London, UK

${ }^{6}$ Imperial College Business School, Imperial College London, London, UK

\section{Correspondence to}

Dr Hutan Ashrafian, Department of Surgery and Cancer, Imperial College London, London SW7

2AZ, UK; hutan@imperial.ac.uk

Received 6 February 2020 Revised 1 July 2020

Accepted 25 July 2020

Published Online First

4 September 2020

A Check for updates

(c) Author(s) (or their employer(s)) 2021. Re-use permitted under CC BY-NC. No commercial re-use. See rights and permissions. Published by BMJ.

To cite: Sounderajah $V$, Patel V, Varatharajan L, et al. BMJ Innov 2021;7:208-216.

\section{ABSTRACT}

The study aims to conduct a systematic review to characterise the spread and use of the concept of 'disruptive innovation' within the healthcare sector. We aim to categorise references to the concept over time, across geographical regions and across prespecified healthcare domains. From this, we further aim to critique and challenge the sector-specific use of the concept. PubMed, Medline, Embase, Global Health, PsycINFO, Maternity and Infant Care, and Health Management Information Consortium were searched from inception to August 2019 for references pertaining to disruptive innovations within the healthcare industry. The heterogeneity of the articles precluded a meta-analysis, and neither quality scoring of articles nor risk of bias analyses were required. 245 articles that detailed perceived disruptive innovations within the health sector were identified. The disruptive innovations were categorised into seven domains: basic science (19.2\%), device $(12.2 \%)$, diagnostics $(4.9 \%)$, digital health $(21.6 \%)$, education $(5.3 \%)$, processes $(17.6 \%)$ and technique (19.2\%). The term has been used with increasing frequency annually and is predominantly cited in North American (78.4\%) and European (15.2\%) articles. The five most cited disruptive innovations in healthcare are 'omics' technologies, mobile health applications, telemedicine, health informatics and retail clinics. The concept 'disruptive innovation' has diffused into the healthcare industry. However, its use remains inconsistent and the recognition of disruption is obscured by other types of innovation. The current definition does not accommodate for prospective scouting of disruptive innovations, a likely hindrance to policy makers. Redefining disruptive innovation within the healthcare sector is therefore crucial for prospectively identifying cost-effective innovations.

\section{INTRODUCTION}

'Innovation', a commonly cited concept in economic and marketing circles, is becoming increasingly recognised as a measurable phenomenon within healthcare. ${ }^{1}$ The father of modern innovation theory, Joseph Schumpeter, described innovation to be 'a historic and irreversible change in the method of production of things'. Given the expansive nature of this definition, we may subcategorise ${ }^{2} 3$ innovation into several broad types: (1) routine/incremental, (2) architectural, (3) radical and (4) disruptive. The concept of 'disruptive innovation', which was originally coined by Clayton Christensen et al ${ }^{4}$ in 1995 , has particularly permeated into academic and clinical healthcare dialect in recent years.

Since the term's inception, Christensen $e t a l^{4}$ has continually reshaped his concept of disruptive innovation in order to accommodate for initial criticisms. In its current and simplest iteration, "disruption' describes the process whereby a smaller company with fewer resources is able to successfully challenge established incumbent businesses'.

Of note, disruption is distinctly characterised by (1) market entrants targeting overlooked segments and (2) gaining traction within said low-end or new-market territories. (3) There is often a slow response to this novel market threat by the incumbent market leaders, who are often focused on evolving their existing product for their established, mainstream customer base. (4) In time, the entrants gain a foothold within the market as they serve a growing demand, often at a lower price.

Non-healthcare-related examples of disruptive innovations include the 

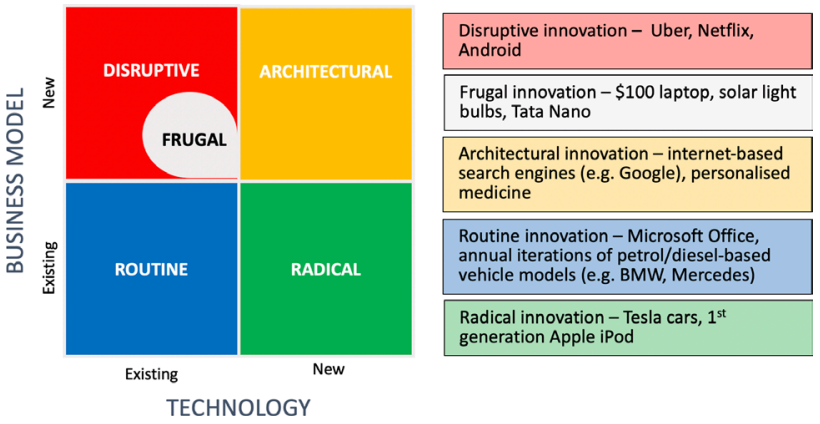

Figure 1 Innovation landscape map (modified from Pisano). ${ }^{5}$

introduction of word processing software (replacing typewriters) and video on-demand services such as Netflix (replacing DVD rental services).

Pisano ${ }^{5}$ notes that disruption specifically occurs at the point at which mainstream customers adopt the market entrant's product in volume. $\mathrm{He}^{5}$ explains (figure 1) that disruption is distinct from other models of innovation as it represents market upheaval through introduction of a technology based on existing technical competencies. Christensen et $a l^{4}$ himself further added to his definition of disruption by highlighting that a disruptor may only be accurately identified retrospectively as opposed to prospectively. He goes on to note that disruptors are based on business models that are distinct to those that are incumbent and that not all disruptions are successful.

Despite the growing use of the concept in medical literature, there are gaps in our understanding of disruptive innovations in a healthcare context. First, there remains an absence of a healthcare sector-specific definition. It is therefore understandable that the use of the term ${ }^{6}$ has been coupled with both imprecision and misuse. This sets a dangerous precedent as misuse within formal peer-reviewed literature muddies the ability for policy makers to identify innovations in their infancy. Accurate identification of successful disruptive innovations is integral to enabling policy makers and healthcare providers to incubate and swiftly disseminate innovations by hastening the adoption-diffusion curve (figure 2), thus reducing their time to institutionalisation. Second, there is no published literature that attempts to identify and compare perceived healthcare disruptive innovations against each other. As such, key innovations in the sector remain entrenched in silos and remain hidden from other work groups that may benefit from them. Lastly, there has been no work to identify either the characteristics of the particular
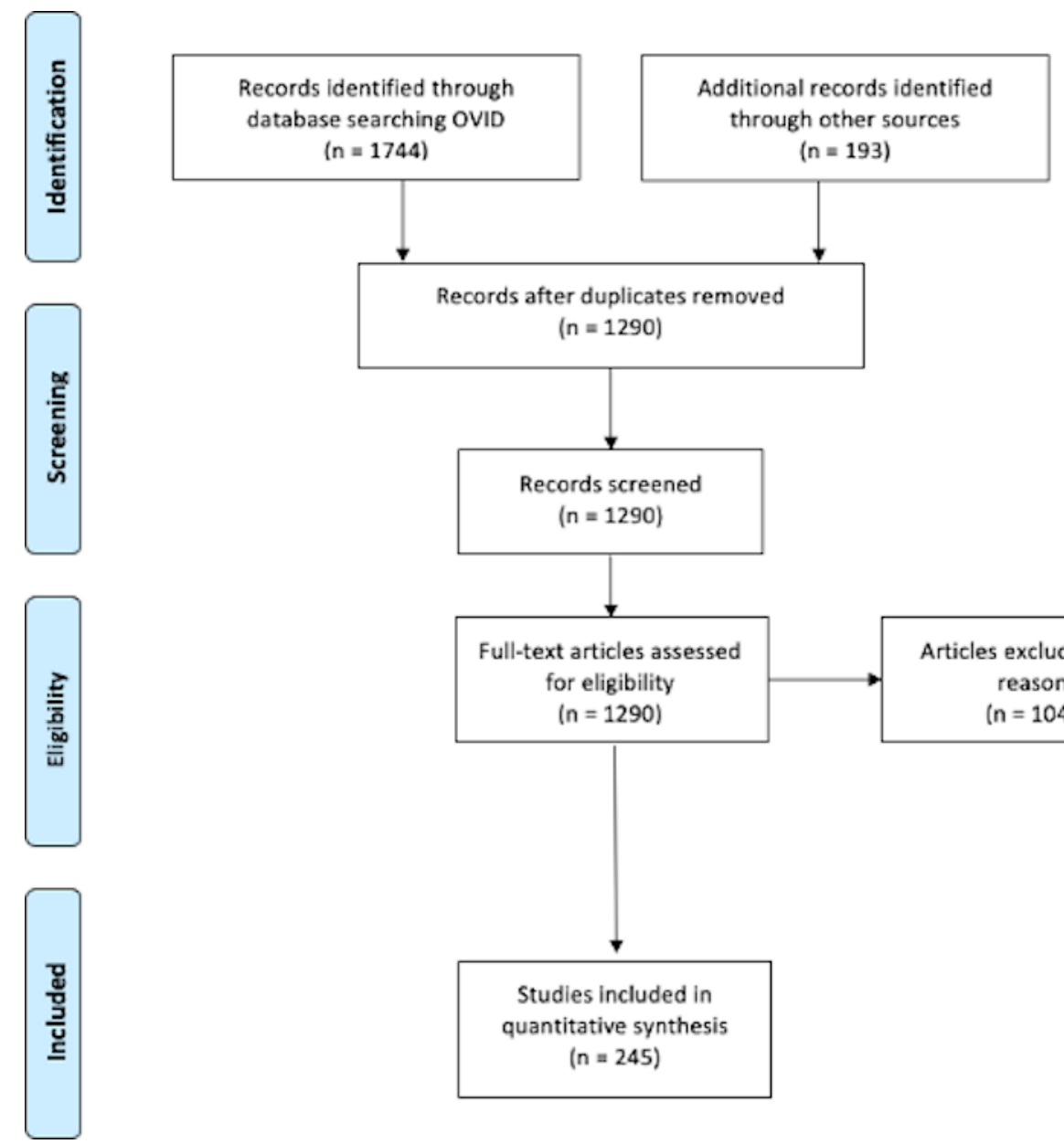

$$
(n=1290)
$$
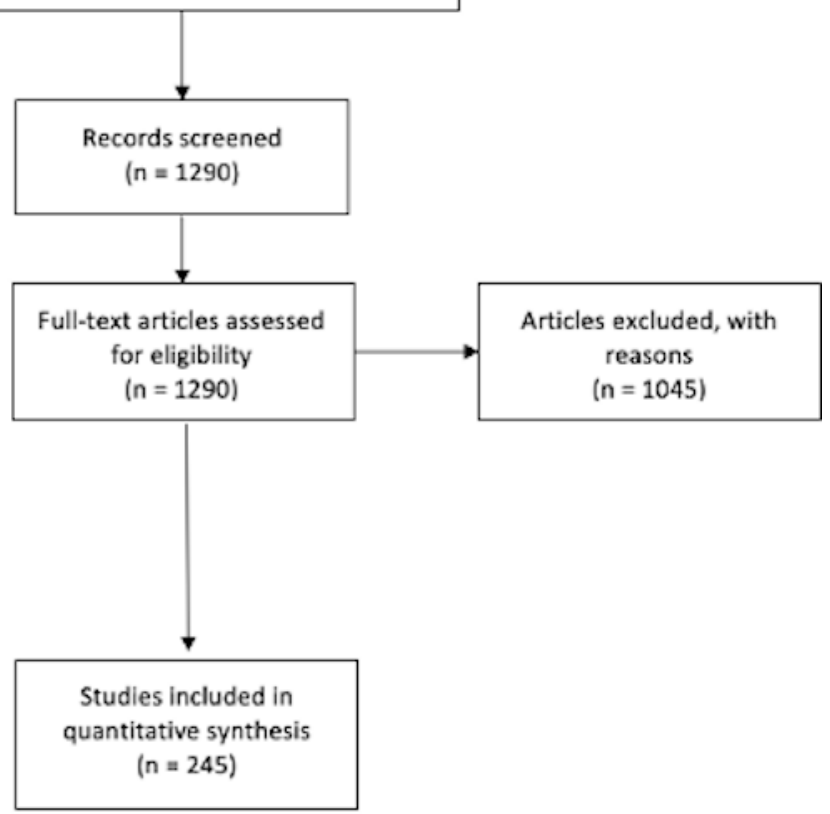

Figure 2 PRISMA diagram detailing study strategy. PRISMA, Preferred Reporting Items for Systematic Reviews and Meta-Analyses. 
sector of healthcare or the geographical location that harbours the most commonly cited disruptive innovations within healthcare. This gap hamstrings our understanding of the context that is required for such innovations to thrive and achieve adoption, a vital learning point for forthcoming implementation strategies. All of these aforementioned issues collectively limit our ability to rapidly identify and incubate disruptive innovations. This, in turn, has a knock-on effect onto the quality of care that health systems are able to provide.

We therefore aimed to study the medical literature to:

1. Systematically review and categorise existing literature regarding the explicit use of the term 'disruptive innovation' or 'disruption' within healthcare.

2. Describe the sectors of healthcare and geographical regions which harbour the highest incidence of perceived disruptive innovation.

3. Critique the usage of the term within the healthcare.

\section{METHODS}

A systematic review following the Preferred Reporting Items for Systematic Reviews and Meta-Analyses (PRISMA) guidelines was conducted. The PubMed, Medline, Embase, Global Health, PsycINFO, Maternity and Infant Care, Health Management Information Consortium and the Cumulative Index to Nursing and Allied Health Literature databases were independently searched from 1948 through to August 2019 by two independent reviewers. Following liaison with a professional librarian, the mutually agreed search strategy was devised (see online supplemental information) for the databases. The 'related articles' function was used with PubMed as a further check of rigour. The last date for this search was 2 August 2019.

\section{Study selection}

Articles were eligible for inclusion if the articles detailed perceived 'disruption' or 'disruptive' innovations within either their title or abstract. Only full peer-reviewed studies and review articles published in the English language were included. Articles were only excluded if they were printed in a language other than English. All potentially eligible studies were retrieved for further evaluation. The search strategy is outlined in online supplemental appendix A. Our PRISMA flow chart is detailed in figure 2 . All shortlisted studies have been listed in online supplemental appendix B.

\section{Data extraction}

Manuscripts were independently extracted (by VS and VP). The data extracted consist of a standardised spreadsheet documenting first author, year of publication, country of publication, study type and type of proposed disruptive innovation (subheadings agreed between authors). All disagreements were resolved through discussion.

\section{Data synthesis and analysis}

For the purposes of the review, both independent reviewers agreed on the categorisation of the healthcare-specific disruptive innovations into seven domains based on those identified in a report on disruptive innovation by the UK's NHS Confederation ${ }^{7}$ :

1. Basic science: this domain relates to novel laboratory (eg, 'omics' disciplines, RNA centric discoveries) and pharmaceutical techniques or discoveries (eg, novel compounds, novel drugs delivery mechanisms).

2. Device: this domain relates to device innovations (eg, point-of-care glucose testing).

3. Diagnostics: this domain relates to either pathological or radiological diagnostic modalities (eg, nerve conduction studies).

4. Digital health: this domain relates to broad use of digital information and communication technology (eg, electronic health records, augmented reality to assist surgery).

5. Education: this domain relates to either the access or provision of education (eg, distance learning).

6. Processes: this domain relates to novel health policy or the reorganisation of structures/health institutions, processes or roles (eg, introduction of nurse practitioners).

7. Technique: this domain relates to the introduction of a novel technique (eg, first instance of endovascular technique in a new specialty-eg, coiling of cerebral aneurysms following the use of established endovascular practice to treat peripheral arterial disease), as opposed to a disruption within a current, well-established technical process (eg, robotic surgery in the field of laparoscopic surgery, which is classed as a device).

Quality scoring using established tools of nonrandomised studies was not applied as the aim of the review was to assess the identification of disruptive innovation in medical publications, as opposed to identifying those identified in randomised/nonrandomised research studies. As a result of the large heterogeneity in publication type,${ }^{8}$ it was agreed by the research group that a quality score would not add additional information for the appraisal of disruptive innovations here.

\section{RESULTS}

Our literature search identified 1937 articles across all bibliographic databases, and 245 articles fulfilled the inclusion criteria (figure 2, online supplemental appendix A). Of the articles, 236 were either secondary review or commentary pieces. Only nine publications were primary research articles. Of the articles, 1045 were excluded during record screening. This was predominantly undertaken as the vast majority of excluded articles used the word 'disruptive' being used as a synonym for troublesome or disorderly, as opposed to the innovation-specific definition. The remainder of the papers were due to the prespecified exclusion criteria. 


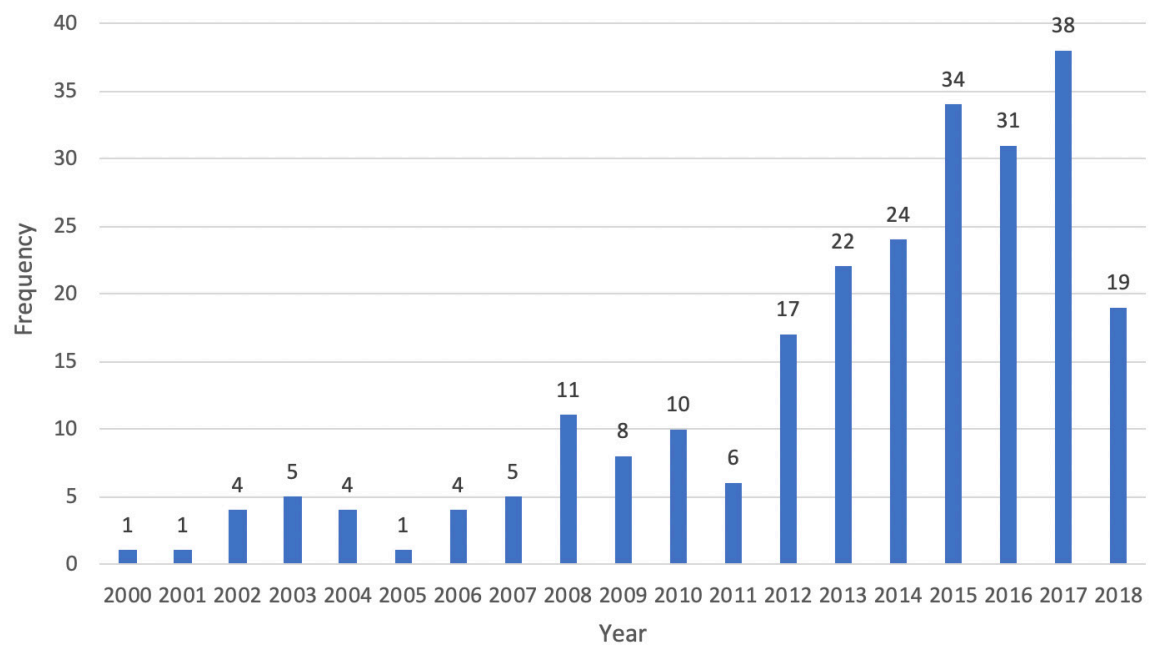

Figure 3 Number of times that disruptive innovation is noted in healthcare literature per annum.

\section{Time}

Given the term's inception was in 1995, the term was first used in medical literature in 2000. Throughout the 2000s, it has been used sparsely, with fewer than 11 references to the concept in any given year. However, particularly since 2012, there has been a marked increase, with a steady yearly rise noted through to 2017 (figure 3).

\section{Geography}

There appears to be strong predilection for the term to be used in high-income settings, as either Europe or the USA is responsible for 229 studies, out of a possible 245 (figure 4). Continents which house the vast majority of low-income countries, namely Asia, Africa and South America, were only responsible for five publications detailing disruptive innovations in total. However, as previously noted, this may also reflect the fact that there may be local dialect-specific translations of the term that are used.

Within the seven domains, we noted the following:

\section{Basic science}

There were 47 papers identified in this field, of which the most cited disruption is the emerging field of 'omics' (26 of 47). Of the omics technologies, genomic technologies were the most commonly cited disruptor in the context of personalised medicine and bespoke oncological treatment. ${ }^{9-11}$ Nanomedicine is also identified as a prominent disruptor. ${ }^{12}$ It is notable that these aforementioned technologies achieved prominence in the 21 st century and there is minimal mention of historically disruptive innovations within the field.

\section{Devices}

There were 30 papers identified in this field, of which the most cited disruptions are the use of threedimensional (3D) printers (5 of 30) and electronic nicotine delivery systems (5 of 30 ). 3D printing has been used for the production of surgical simulation models, ${ }^{13}$ through to organs for surgical cases. ${ }^{14}$

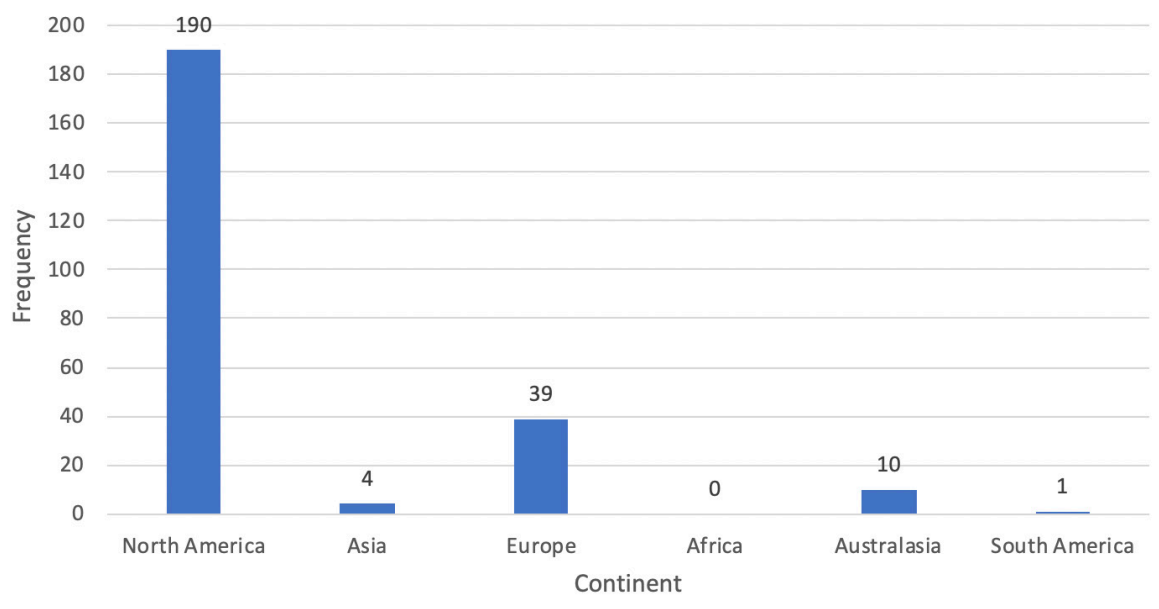

Figure 4 Continent of origin of publications detailing a disruptive innovation. 


\section{Diagnostics}

There were 12 papers noted in this field, of which the most cited disruption is the use of CT imaging ( 3 of 12). Of note, diagnostic innovations which primarily relied on a digital health platform, such as remote monitoring, were considered to be classified as part of the digital health domain.

\section{Digital health}

There were 53 papers noted in this field, of which the most cited disruption is on the subject of mobile health (mHealth) (25 of 53), particularly in the context of inpatient care, ${ }^{15}$ outpatient self-care ${ }^{16}$ and telemedicine. ${ }^{17}$ Health informatics, particularly the analysis of clinical big data sets, ${ }^{18}{ }^{19}$ was also highlighted prominently. These examples highlight that many digital health disruptive innovations encroach on other domains, such as diagnostics and processes.

\section{Education}

There were 13 papers noted in this field, of which the most cited disruption is distance learning, predominantly through internet-based modules. ${ }^{20}$ This is the least cited domain of healthcare disruptive innovations.

\section{Processes}

There were 43 papers noted in this field, of which the most cited disruption is the establishment of retail clinics, predominantly in North America (6 of 43). In addition, the expansion of nursing responsibilities (eg, undertaking cardiopulmonary resuscitation ${ }^{21}$ and adopting nurse practitioner roles ${ }^{22}$ ) is highlighted.

\section{Technique}

There were 47 papers noted in this field, of which the most cited disruptions are related to endovascular surgery. References to this technique range from broad pan-specialty references to specialty-specific examples (eg, neurovascular surgery ${ }^{23}$ ).

Cumulatively across these domains, the following are the five most cited (figure 5) disruptive innovations:

1. Digital health: telemedicine.

2. Digital health: mHealth.
3. Basic science: 'omics' fields.

4. Processes: retail clinics.

5. Digital health: health informatics.

\section{DISCUSSION}

This systematic review broadly addresses several aforementioned knowledge gaps regarding the dissemination and use of the concept of 'disruptive innovation' within the healthcare industry. The results suggest that while there appears to be penetration of the term across broad healthcare sector-related domains, this is a relatively recent phenomenon, with a striking skew towards publications in high-income countries.

Since 1995, there have been 245 published references to disruptive innovation within the healthcare literature globally, and of these 185 have been published since 2012 (figure 3). This recent surge in citations of the concept could represent two contrasting phenomena. The rise in published mentions of the concept may be proportionate to the level of disruptive innovations that have occurred during the commensurate timeframe. However, what is more likely is that the term has been imprecisely used, perhaps partly due to the lack of an objective definition readily available in the healthcare literature. Therefore, the literature has captured both accurate and inaccurate uses of the term. It is also likely that this rise in literature citations mirrors the increasing use of the term in more mainstream sources.

We have also demonstrated that the use of the term 'disruptive innovation' is concentrated in publications originating from North America and Europe (figure 4). Given the origin of the term (Christensen is based in Boston, Massachusetts, USA), the skew towards North America is understandable. We note that the health literature from these high-income nations tends towards the use of the term 'disruption', whereas there is evidence from low-income to middle-income health systems of being more open to the concept of frugal innovation. ${ }^{24}$ This may be a contributing factor to a lack of published disruptive

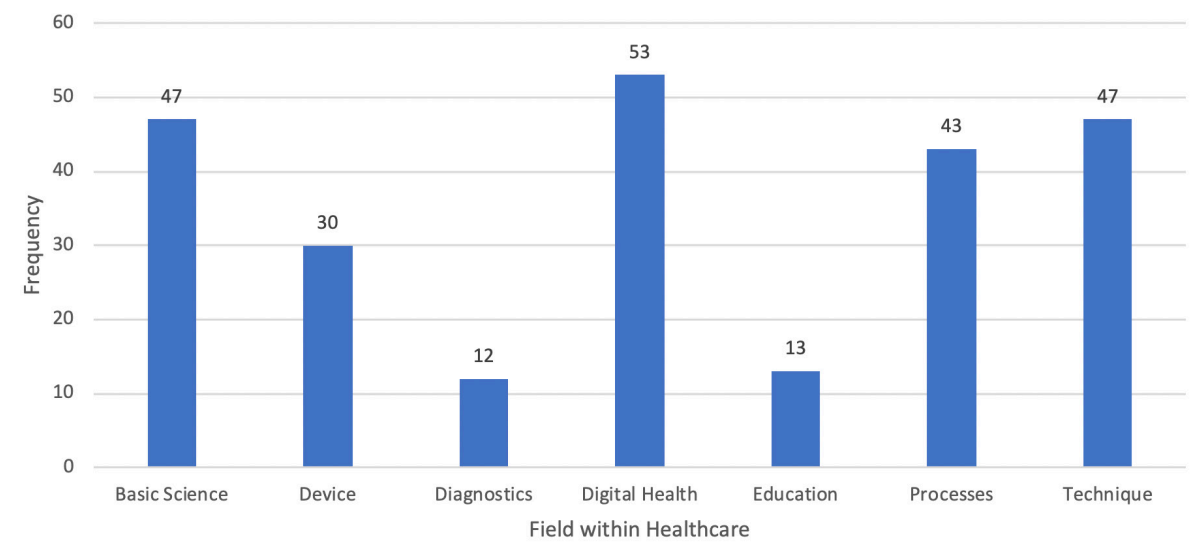

Figure 5 Most cited categories of disruptive innovation within healthcare. 
innovations in Africa, South America and Asia, respectively, which have produced only $2.4 \%$ of the captured literature. ${ }^{25}$ However, we do note that the geographical spread of the studies may be skewed by the exclusion criteria, which eliminated the studies published in languages other than English. Although this criterion has been imposed in order to capture specific uses of the term 'disruption' as meant by Christensen et al, ${ }^{4}$ this admittedly does not accommodate for local dialect translations of the term.

It is noteworthy that there is significant variation in the degree in which each healthcare domain is affected by disruptive innovation. The most cited disruptive innovations (figure 5) are predominantly digital health and basic science-based; mHealth, telemedicine, health informatics and 'omics' centric innovations all featuring within the top five most cited disruptors. In the domains related to digital health, techniques, devices, processes and basic science, the concept is cited with near equal frequency, with each domain consisting of between $16 \%$ and $22 \%$ of mentions. The skew towards digital health, devices and basic science being labelled as disruptive innovations may stem from the fact that the concept of an innovation is often used interchangeably with invention by many. In contrast, the fields of education and diagnostics made up only $5 \%$ and $2 \%$ of the articles. Education in particular may have suffered from reduced attempts at disruptive innovation as it may be seen as a less lucrative and appealing avenue to dedicate technology towards. Moreover, as education may not involve the introduction of a paradigm shifting technology, more subtle disruptors (eg, introduction of distance learning) may go relatively unidentified.

However, we do note that there are several examples of appropriately defined disruptive innovations, particularly with respect to the process innovation of single specialty services, which are associated with highly lean workforces and cheaper technology in order to drive down costs and supplant the market incumbents. Such examples include the Shouldice Hospital in Canada, Coxa Hospital in Finland, ${ }^{26}$ as well as Aravind Eye Care and Narayana Health Hospitals, which are both based in India. ${ }^{27}$

Despite such instances of appropriate identification, mislabelling of disruption is rife across all seven domains within the literature. Repeated offenders include the mislabelling of endovascular surgery, as an entire field, as a disruptor. This is more accurately described as a radical innovation, using Pisano's categorisation $^{5}$ (figure 1), as it is the introduction of a novel technology within the field of surgery, a well-defined market. Moreover, another clear misuse is to suggest that the application of omics technologies in the field of personalised medicine is a disruptive innovation. This is more accurately described as an architectural innovation, as it refers to the use of a novel technology within an unprecedented field (figure 1).
Across the 245 included studies, the following are some of the most fundamental errors and deviations from Christensen's ${ }^{4}$ principles:

1. Describing the technologies themselves as disruptive innovations, as opposed to the process of market upheaval: a prominent example of this flaw is describing the software offering a telemedicine service as a disruptive innovation. Each brand and variant of telemedicine is notably individually cited in the literature as a disruptive innovation in itself, with undue emphasis on the product as opposed to the process by which it fulfils an underserved market need. Minimal attention is given to the process innovation associated with this service, through which there is associated reorganisation of outpatient services and resources.

2. Describing theoretical market effects of products yet to reach the open market as a 'prospective' disruptive innovation: disruptive innovation, in its truest sense, may only be truly labelled as such retrospectively. Perceived potential success of a technology does not necessarily make for a disruptive innovation. This error is evident by the multiple references to artificial intelligence (AI) as being a burgeoning disruptive innovation. Although there have been prominent applications of AI within academic medical research, ${ }^{28-31}$ there is no guarantee that it will achieve disruption within its intended market. Moreover, labelling the broad field AI as a disruptive innovation, a field of computer science as opposed to a soft technology with a singular purpose, deviates from Christensen's ${ }^{4}$ definition of focusing on distinct market sectors within which a technology acts.

3. Describing innovations as 'disruptive' which would be more accurately described as 'sustaining innovation': one such example is the concept of drug eluting stents. Drug eluting stents have shown superiority in preventing coronary artery restenosis in multiple trials in comparison with bare metal stents ${ }^{32}$ and have subsequently been erroneously been labelled as a disruptive innovation. Given that this technology was based on the original innovation of bare metal stents, which had replaced the role of open surgery in many cases, it can be argued that drug eluting stents are more accurately described as an incremental innovation.

4. According to Christensen et al, ${ }^{4}$ disruptive innovations eventually always supplant the incumbent market leader: long-standing coexistence is not classically in keeping with true disruption. Therefore, the suggestion that laparoscopic surgery, as an entire practice, is a disruptive innovation is misleading as there is still huge scope for open surgery globally. This error once again demonstrates the flaw in labelling an entire practice as a disruptive innovation. It is more accurate to state that a particular laparoscopic practice, such as laparoscopic cholecystectomy, is a disruptive innovation within the field of elective surgery as it has almost entirely supplanted the open surgical technique for benign gallbladder disease. This is not the case for hernial disease, for example.

Despite dubious accuracy in the use of the term in the literature, it does appear that there is a breadth in the use of the concept across domains, as previously mentioned, which suggests that there is a lay awareness of the term among the healthcare sector 
audience. The concept of disruptive innovation has been used in themes ranging from policy literature in describing the Affordable Care Act, the most fundamental restructuring of healthcare provision in the USA since $1965,{ }^{33}{ }^{34}$ through to its more conventional application to a technology, such as a subcutaneous implantable cardioverter defibrillator ${ }^{35}$ within the domain of medical devices. Both examples have been labelled as disruptors within their respective field, yet both are vastly different in their effect and scope. As such, when attempting to apply a singular definition across disciplines, it is important to recognise that not all disruptive innovations are equal in overall impact and reach. Therefore, each individual innovation warrants contextual assessment within its own field, which suggests that one should not rely on a singular catchall mechanism or recognised metric to identify these innovations.

It has been previously noted that the use of the term 'potential disruptor' is an inaccuracy as per Christensen's ${ }^{4}$ principles. Two such examples are that of $\mathrm{AI}^{36}$ and cryptography, ${ }^{37}$ both of which have been recently labelled by opinion leaders ${ }^{38-40}$ as being impending pan-industry disruptors. Although this constitutes imprecise use of the concept, it highlights that Christensen's ${ }^{4}$ definition, in its current iteration, is too rigid for policy makers to use in a constructive fashion. His definition does not accommodate for the identification of prospective disruptive innovations as it is more concerned with retrospective identification. In contrast to the current paradigm of focusing on disruptors retrospectively, ${ }^{41}$ there is a strong argument to state that there is an economic and ethical obligation for policy makers to accurately identify disruptive innovations prospectively. This would aid in technology intelligence initiatives as previously noted. In selecting candidate innovations during an earlier phase of their life-cycle, they can be appropriately incubated, disseminated and translated, ultimately delivering maximal societal benefit within a short timeframe. This in turn could result in a reduction of the time for the diffusion of healthcare innovations (often cited as 17 years $^{42}$ ). However, caution must also be displayed when choosing novel innovations as disruptive. Overly zealous contemporaneous labelling of disruptive innovations may lead to the incubation of technologies, which has long-term deleterious effects. As such, each innovation should be considered by its own individual merits in addition to its individual risk profile.

Adaptation of the definition may also help pick many historic disruptive innovations that are now commonplace that have not been noted within the literature. There is minimal reference in the literature to the rise of laparoscopic surgical techniques and endoscopic procedures as successful disruptive forces within their respective fields. By not highlighting successful historic disruptive innovations, the healthcare sector is at risk of not learning from past successful diffusion-adoption strategies.

It can be argued that the lack of a tailored, pragmatic definition of disruptive innovation in healthcare may, in part, explain why there appears to be lengthy and variable times to recognise disruption. A 'realworld' example of this is the laparoscopic approach to cholecystectomies, long seen as a disruptor to surgical practice. The first laparoscopic cholecystectomy was performed in 1985 in Germany. ${ }^{43}$ Despite welldocumented benefits with respect to both morbidity and mortality, the widespread adoption of this technique lagged until the late 1990s in high-income countries. Similarly, the first endovascular aortic aneurysm repair was performed in 1990 to much acclaim, ${ }^{44}$ however was not part of standard practice until the 2000s. Further historic examples which notably failed to initially identify as burgeoning disruptions include the citric acid cycle, ${ }^{45}$ the invention of the immunoassay ${ }^{46}$ nuclear magnetic resonance ${ }^{47}$ and the PCR, ${ }^{48}$ all of which have since warranted Nobel Prize awards in their respective fields following their initial peer review journal rejection, further highlighting the issue with contemporaneous recognition of innovation in healthcare.

\section{CONCLUSION}

Disruptive innovation is a term that has diffused into the healthcare industry, but there is widespread ambiguity in the use of the term. It may have become a victim of its own mainstream success. Poor identification can lead to poor understanding of the characteristics and potential of an innovation. This in turn can contribute to delay in its translation into tangible economic and health outcomesbased benefits because we fail to understand the potential barriers to adoption and ways to overcome them. We suggest that a more precise healthcare-specific definition, achieved through an expert consensus process, would be a precursor to better identification of potentially beneficial disruptive innovations and shortening their journey to translation and diffusion.

Twitter James Barlow @jgbarlow2010

Acknowledgements Infrastructure support was provided by the NIHR Imperial Biomedical Research Centre.

Contributors HA, LH, AD and VS planned the systematic review. VS and VP conducted the search. LV, JS and PN conducted the analysis. All authors contributed equally to the writing of the paper. HA, JB and AD undertook the senior review of the work on completion of the manuscript preparation. HA is responsible for the overall content of the study.

Funding The authors have not declared a specific grant for this research from any funding agency in the public, commercial or not-for-profit sectors.

Competing interests None declared.

Patient consent for publication Not required.

Provenance and peer review Not commissioned; externally peer reviewed.

Data availability statement Data are available in a public, open access repository. All data, extracted from peer-reviewed 
publications, are readily available through traditional means (PubMed, Google Scholar).

Open access This is an open access article distributed in accordance with the Creative Commons Attribution Non Commercial (CC BYNC 4.0) license, which permits others to distribute, remix, adapt, build upon this work non-commercially, and license their derivative works on different terms, provided the original work is properly cited, appropriate credit is given, any changes made indicated, and the use is non-commercial. See: http://creativecommons.org/ licenses/by-nc/4.0/.

ORCID iD

Viknesh Sounderajah http://orcid.org/0000-0002-4595-8402

\section{REFERENCES}

1 Fagerberg J, Mowery D, Nelson RR. Innovation: a guide to the literature, 2003. Available: http://tikpc51.uio.no/teari/teari.htm

2 Satell G. The 4 types of innovation and the problems they solve. Harv Bus Rev 2017;11:2-9.

3 Barlow J. Managing Innovation in Healthcare [Internet]. World Scientific (Europe), 2017. Available: http://www. worldscientific.com/worldscibooks/

4 Christensen CM, Raynor M, McDonald R. What is disruptive innovation? Harvard Buisness Rev 2015:1-11.

5 Pisano GP. You need an innovation strategy. Harv Bus Rev 2015.

6 William M. Disruptive Innovation in Healthcare: What to Expect in 2016. Samsung [Internet], 2016. Available: https:// insights.samsung.com/2016/04/05/disruptive-innovation-inhealthcare-what-to-expect-in-2016/

7 The NHS Confederation. Disruptive innovation What does it mean for the NHS? [Internet], 2008. Available: www. debatepapers.org.uk

8 Howick J, Chalmers I, Lind J, et al. Oxford Centre for Evidence-Based Medicine 2011 Levels of Evidence [Internet]. Available: http://www.cebm.net/index.aspx?o $=5653$

9 Tursz T, Bernards R. Hurdles on the road to personalized medicine. Mol Oncol 2015;9:935-9.

10 Parkinson DR, Ziegler J. Educating for personalized medicine: a perspective from oncology. Clin Pharmacol Ther 2009;86:23-5.

11 Kittaneh M, Montero AJ, Glück S. Molecular profiling for breast cancer: a comprehensive review. Biomark Cancer 2013;5:BIC.S9455-70.

12 Ptak K, Farrell D, Panaro NJ, et al. The NCI alliance for nanotechnology in cancer: achievement and path forward. Wiley Interdiscip Rev Nanomed Nanobiotechnol 2010;2:45060.

13 Hui DS, Lee R. Scan, plan, print, practice, perform: a disruptive technology? J Thorac Cardiovasc Surg 2017;153:141-2.

14 Yoo S-S. 3D-Printed biological organs: medical potential and patenting opportunity. Expert Opin Ther Pat 2015;25:507-11.

15 Nguyen HH, Silva JNA. Use of smartphone technology in cardiology. Trends Cardiovasc Med 2016;26:376-86.

16 Swendeman D, Ramanathan N, Baetscher L, et al. Smartphone self-monitoring to support self-management among people living with HIV. JAIDS J Acquir Immune Defic Syndr 2015;69:S80-91.

17 Rosen R. Are disruptive innovators in GP provision strengthening or weakening the NHS? BMJ 2017;359:j5470.

18 Felker GM, Ahmad T. Reclassifying heart failure: time for disruptive innovation? Eur J Heart Fail 2015;17:879-80.

19 Kricka LJ. Emerging and disruptive technologies. EJIFCC 2016;27:253-8.
20 Thompson CJ. Disruptive innovation. Clin Nurse Spec 2016;30:238-41.

21 Edmonson C, Klacman A, Tippy J. Nurse leaders as disruptive Innovators in cardiopulmonary resuscitation competency. Nurse Lead 2016;14:191-4.

22 Edmunds M. Consider yourself a "disruptive innovator". Nurse Pract 2002;27:15.

23 Rosenwasser RH, Lang M, Tjoumakaris S, et al. Disruptive innovation in neurovascular disease. Neurosurgery 2017;64:78-82.

24 Basu R, Banerjee P, Sweeny E. Frugal innovation: core competencies to address global sustainability. J Manag Glob Sustain 2013;1:63-82.

25 Bhatti Y, Taylor A, Harris M, et al. Global lessons in Frugal innovation to improve health care delivery in the United States. Health Aff 2017;36:1912-9.

26 Disrupting The Hospital Business Model [Internet]. Available: https://www.forbes.com/2009/03/30/hospitals-healthcaredisruption-leadership-clayton-christensen-strategy-innovation. html\#6ba6ab0f6512

27 Castano R. Towards a framework for business model innovation in health care delivery in developing countries. BMC Med 2014;12:233.

28 Rajkomar A, Oren E, Chen K, et al. Scalable and accurate deep learning with electronic health records. NPJ Digit Med 2018;1:18.

29 Mori Y, Kudo S-E, Misawa M, et al. Real-Time use of artificial intelligence in identification of diminutive polyps during colonoscopy: a prospective study. Ann Intern Med 2018;169:357.

30 Komorowski M, Celi LA, Badawi O, et al. The artificial intelligence clinician learns optimal treatment strategies for sepsis in intensive care. Nat Med 2018;24:1716-20.

31 De Fauw J, Ledsam JR, Romera-Paredes B, et al. Clinically applicable deep learning for diagnosis and referral in retinal disease. Nat Med 2018;24:1342-50.

32 Stefanini GG, Holmes DR. Drug-Eluting coronary-artery stents. N Engl J Med 2013;368:254-65.

33 Blumenthal D, Abrams M, Nuzum R. The Affordable care act at 5 years. N Engl J Med 2015;372:2451-8.

34 Oberlander J. Long time coming: why health reform finally passed. Health Aff 2010;29:1112-6.

35 Saarel EV. Sustaining and Disruptive Innovation in Clinical Electrophysiology: The Subcutaneous Implantable Cardioverter-Defibrillator in the Young. JACC Clin Electrophysiol 2017;3:1507-9.

36 Hamet P, Tremblay J. Artificial intelligence in medicine. Metabolism 2017;69S:S36-40.

37 Mettler M. Blockchain technology in healthcare: the revolution starts here. 2016 IEEE18th Int Conf e-Health Networking, Appl Serv Heal, 2016:16-18.

38 Deloitte Center for Health Solutions. Robots, AI, and cognitive analysis: How technology is disrupting hospitals around the globe [Internet]. Available: http://blogs.deloitte.com/cent erforhealthsolutions/robots-ai-cognitive-analysis-technologydisrupting-hospitals-around-globe/

39 Gretton C, Honeyman M. The digital revolution. The King's Fund [Internet], 2016. Available: https://www.kingsfund.org.uk/ publications/eight-technologies-will-change-health-and-care

40 McKinsey \& Company. Artificial intelligence: The time to act is now [Internet]. Available: https://www.mckinsey. com/industries/advanced-electronics/our-insights/artificialintelligence-the-time-to-act-is-now

41 Hickman C, Raia C. Incubating innovation. J Bus Strategy 2014. 
42 Slote Morris Z, Steven Wooding B, Jonathan Grant B. The answer is 17 years, what is the question: understanding time lags in translational research. Available: http://journals.sagepub.com

43 Reynolds W. The first laparoscopic cholecystectomy. JSLS J Soc Laparoendosc Surg 2001;5:89-94.

44 Parodi JC, Palmaz JC, Barone HD. Transfemoral intraluminal graft implantation for abdominal aortic aneurysms. Ann Vasc Surg 1991;5:491-9.

45 Krebs HA. The citric acid cycle: a reply to the criticisms of F. L. Breusch and of J. Thomas. Biochem J 1940;34:460-3.
46 Yalow RS, Berson SA. Immunoassay of endogenous plasma insulin in man. J Clin Invest 1960;39:1157-75.

47 Ernst RR, Anderson WA. Application of Fourier transform spectroscopy to magnetic resonance. Rev Sci Instrum 1966;37:93-102.

48 Mullis K, Faloona F, Scharf S, et al. Specific enzymatic amplification of DNA in vitro: the polymerase chain reaction. Cold Spring Harb Symp Quant Biol 1986;51 Pt 1:263-73. 